\title{
Monitoring the ESLEA UKLight network
}

\section{Barney Garrett ${ }^{1}$}

The University of Edinburgh

James Clerk Maxwell Building, Mayfield Road, Edinburgh. EH54 6TD. UK

E-mail: barney.garretteed.ac.uk

The eScience applications taking part in the ESLEA project aim to utilise the switched light paths offered by UKLight to deliver massive datasets and prove bandwidth intensive data streaming technologies. For debugging, analysis and reporting, the applications need to be able to monitor their bandwidth utilisation. This paper looks at the methods that could be used and the implementation of those technologies within the UKLight infrastructure.

Lighting the Blue Touchpaper for UK e-Science - Closing Conference of ESLEA Project The George Hotel, Edinburgh, UK

26-28 March, 2007

\footnotetext{
1 Speaker
} 


\section{Introduction}

The ESLEA[1] project's objective is to utilise the switched lightpath network, UKLight[2], provided by UKERNA[3] to support the data transfer requirements of the eScience community. In order to assess the success of the project and to be able to provide reporting data the collection of traffic throughput and utilisation is essential. The project has UKLight links terminating in the UK at UCL, Lancaster, Manchester and Edinburgh, and due to the nature of UKLight it is only possible for us to monitor the network traffic at these points [Figure 1]. The monitoring has been implemented in several stages, with each stage attempting to improve the usefulness of the collected data.

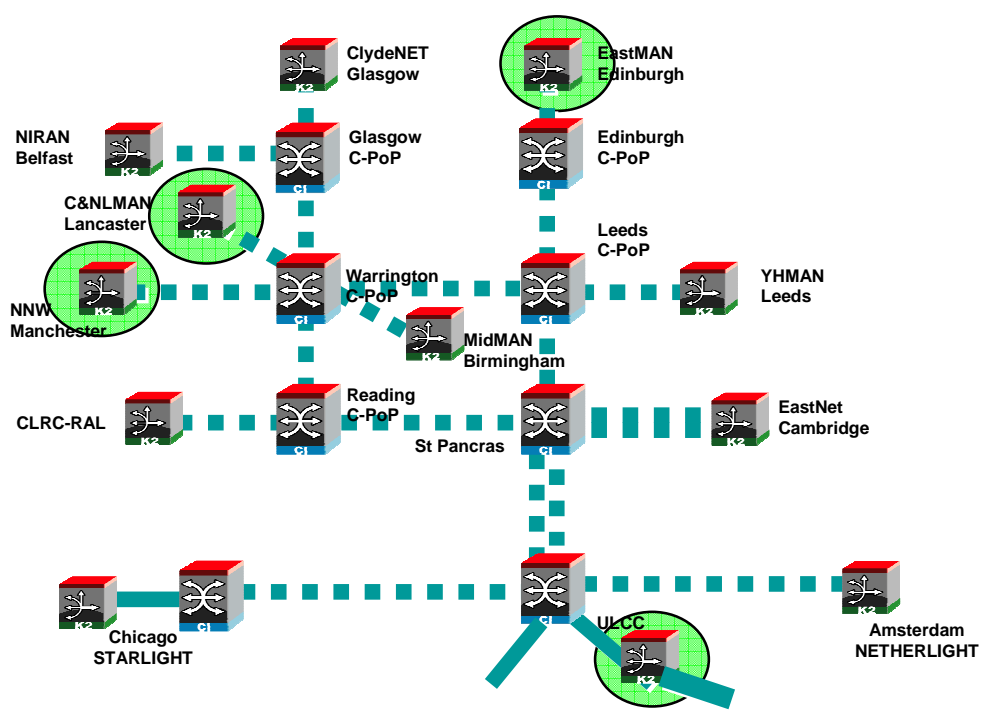

Figure 1

\section{MRTG}

The Multi Router Traffic Grapher (MRTG)[4] is a tool to monitor the traffic load on network links. MRTG generates HTML pages containing images which provide a visual representation of this traffic [Figure 2]. The first iteration of our "monitoring system" was to install an MRTG monitoring server attached to the project routers at each of the sites. This gave us basic utilisation monitoring capabilities for each of the UKLight links. 


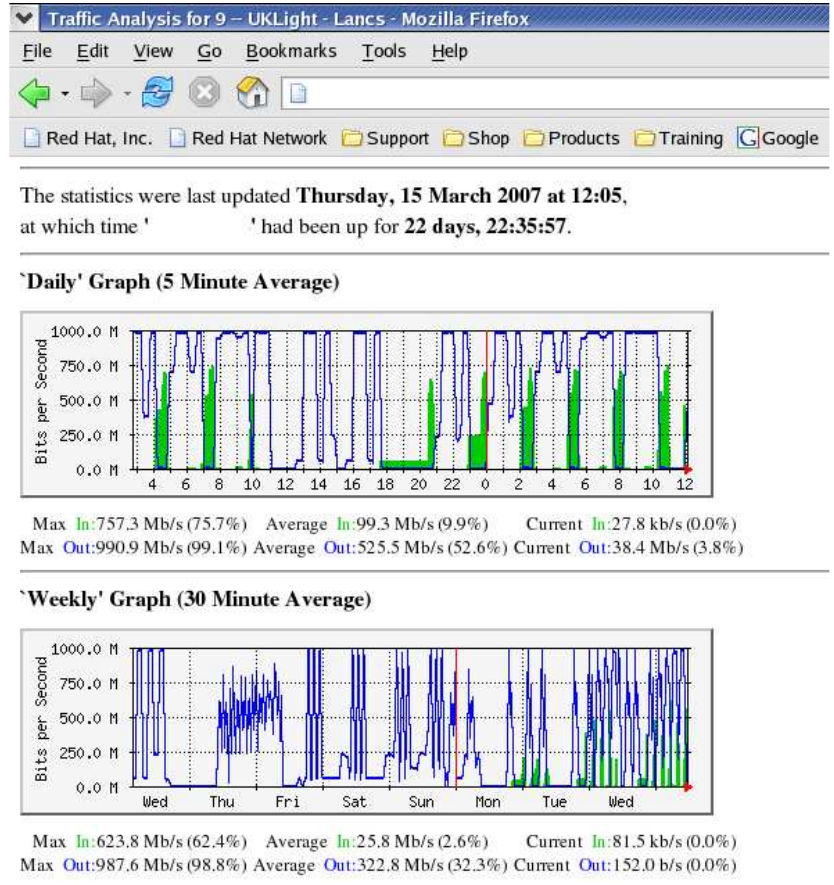

Figure 2

\section{Weathermap}

A "weathermap"[5] style of visualisation was made to bring all the MRTG resources together into a simple and easily understood interface. It acquires the information provided by the MRTG system and displays it on a map to represent the logical topology of the ESLEA project UKLight links [Figure 3][6].

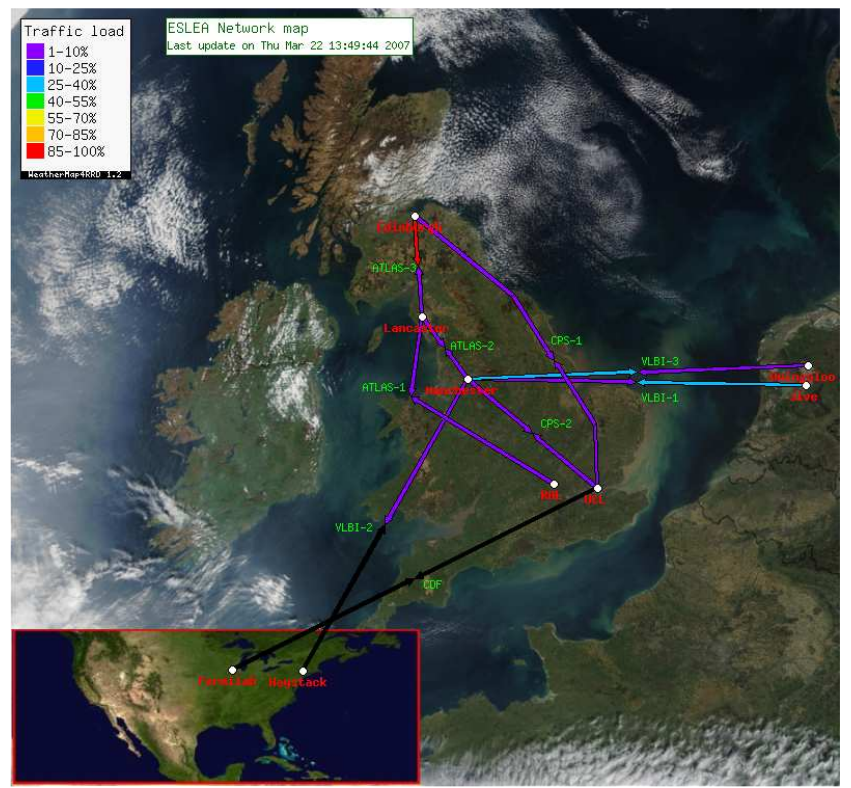

Figure 3 


\section{RRDTool and PerfSonar}

The MRTG system is limited to taking samples every 5 minutes, and averages the data down into longer periods when looking at historic data which can lead to misleading figures. To overcome these limitations a Round Robin Database (RRD)[7] was then deployed. This is a tool set that allows the storage of time-series data in an efficient and systematic manner and provides tools for analysing that data. Using RRD we increased the sample collection rate to every 60 seconds, and increased the accuracy of the figures when looking at historic data.

The PerfSonar project[8], the result of a joint collaboration between ESnet, Geant2, Internet 2 and RNP, is a web services infrastructure for network performance monitoring that provides services for publishing the data stored within these RRD files. By utilising the PerfSonarUI[9] it is possible to interrogate these web services to dynamically produce graphs similar to the ones produced by MRTG showing inbound and outbound throughput [Figure 4] but with more granularity and other additional features such as the ability to zoom in on areas of particular interest.

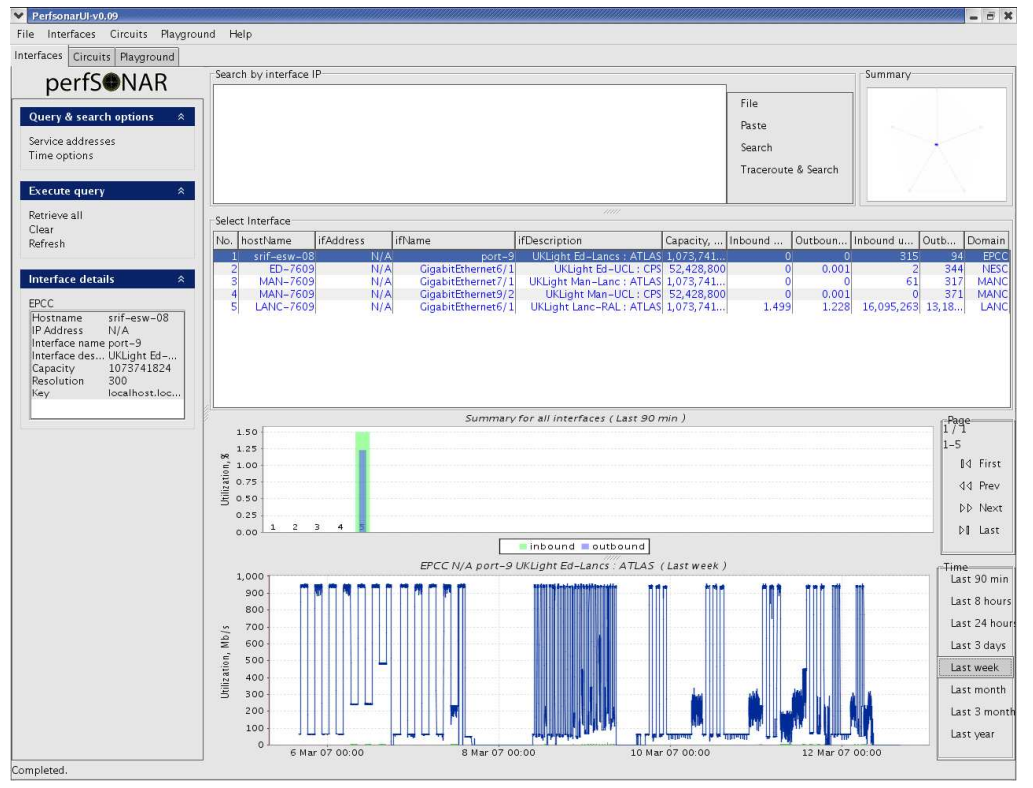

Figure 4

\section{Summary}

All three tools can provide useful utilisation metrics to a project assuming appropriate access to the networking equipment can be arranged. Both MRTG and the Weathermap tools were easy to install and configure but are limited in their resolution. The Weathermap in particular provides an easy to digest view where there are a larger number of connections being monitored. PerfSonar is more suited to being deployed by the network service providers rather than on a project level as is has a rather convoluted installation and configuration process, it is however by far the most advanced and flexible of the systems that we used. 


\section{References}

[1] ESLEA http://www.eslea.uklight.ac.uk

[2] UKLight http://www.uklight.ac.uk

[3] UKERNA http://www.ukerna.ac.uk

[4] MRTG http://oss.oetiker.ch/mrtg

[5] Weathermap4php http://weathermap4rrd.tropicalex.net/index.php

[6] ESLEA Weathermap http://www.eslea.uklight.ac.uk/weathermap

[7] RRD Tool http://oss.oetiker.ch/rrdtool

[8] PerfSonar http://www.perfsonar.net

[9] PerfSonarUI http://perfsonar.acad.bg 